\title{
Approach and avoidance effects of central stimulation: An exploration of the pigeon fore- and midbrain ${ }^{1,2}$
}

\author{
IRVING J. GOODMAN, Center for Brain \\ Research. University of Rochester, \\ Rochester. N.Y. 146273
}

Chronically indwelling multiple electrodes were employed to investigate the reinforcement effects of unipolar electrical brain stimulation (ESB) at foreand midbrain sites in the pigeon (Columba livia). Using a place-preference task, approach sites, which were fewest in number $(7.1 \%)$, were identified in the telencephalon (paleostriatum and archistriatum), with none noted in di- or mesencephalon: avoidance sites (35.7\%) were identified in tel-, di-, and mesencephalon. Changes in current intensity produced shifts from neutral to approach effects at some sites, neutral to avoidance effects at others, and no shifts at still others. Results were discussed in relation to relevant mammalian and avian findings.

The reinforcing effects of electrical brain stimulation (ESB) have been reported both in terms of positive (approach behavior) (Olds \& Milner, 1954) and negative effects (avoidance behavior) (Delgado et al, 1954) with the characteristics of these effects having been most thoroughly investigated in mammals. Among nonmammals, reinforcement effects of ESB have been observed in fishes (Boyd \& Gardner, 1962) and birds: chicks (Andrew, 1967) and pigeons (Goodman \& Brown, 1966; Macphail, 1967).

A variable considered most critical in affecting the reinforcement valence of ESB is the locus of stimulation. A review of results from a number of mapping studies in different mammalian species (reviewed by Olds \& Olds, 1963) shows that approach and avoidance responses to ESB are attainable from separable points distributed within fore- and midbrain structures.

Because of the rather sparse information concerning the distribution of central reinforcement points in the bird, the present study has attempted to explore a variety of fore- and midbrain structures in the pigeon with electrical stimulation and to assess the behavioral consequences.

Fig. 1. Mean stimulation percentages at different current intensities. shuttlebox compartment containing the illuminated signal light; signal-light illumination (2.min duration) was alternated between compartments. In each daily session, Ss had an opportunity to receive as many as $1,2000.5-\mathrm{sec}$ shocks with the performance for the last 600 constituting the reported data. The criteria adopted for reinforcement effects were based upon the per cent of available ESBs actually received: $65 \%$ and above was judged approach behavior, $35 \%$ and below was judged avoidance (or escape) behavior, and scores between these percentages were considered neutral. Ss were tested at several current intensities $(0.2,0.4,0.6$, 0.8 , and $1.0 \mathrm{~mA}$ ) at each electrode point; only one brain point in a bird was stimulated at a single intensity during a session with each intensity tested at each point in two or more sessions.

Following all behavioral tests, Ss were sacrificed with an overdose of sodium pentobarbital. Frozen brain sections (50 microns) were cut and alternately stained for fibers and cell bodies. Slide preparations were examined under a light microscope in order to determine electrode tip locations.

\section{RESULTS}

Approach Effects

of experimental Recording and programming automatically by electromechanical equipment.

A place preference test was used to determine the reinforcement effects of ESB at 70 electrode placements. Ss received a 0.5 -sec train of ESB once every $4 \mathrm{sec}$ only when they were in the
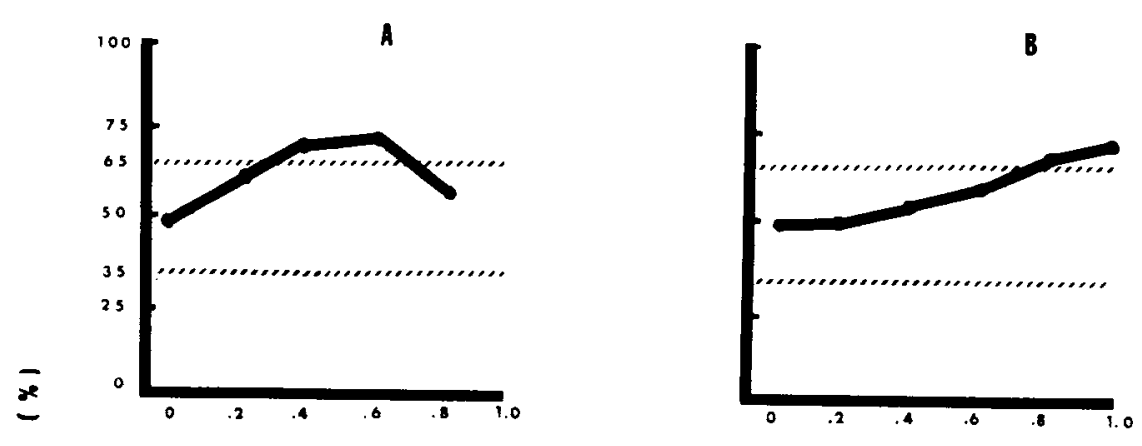

Of the 70 brain points stimulated, five, or $7.1 \%$, showed approach effects. However, such effects did not occur at all intensities tested at the same placement; an example of one bird's performance is illustrated in Fig. 1A, in which there appeared to be optimal intensities for $\infty$

$\infty$ w 
approach effects, on either side of which effects were neutral. Another intensity-performance curve is seen in Fig. 1B, in which approach effects increased with higher currents. Both of these effects resulted from stimulation in paleostriatum in two different Ss.

Avoidance Effects

Points yielding avoidance behavior numbered 25 , or $37.5 \%$ of the total tested. Similarly, avoidance performance appeared also to be a function of stimulus intensity; current increases beyond a critical level generally tended to increase the level of avoidance behavior (Fig. 1C). Within the range of currents employed, no stimulated point showed both positive and negative reinforcement effects. On the other hand, two birds with multiple electrode placements showed one approach, one avoidance, and two neutral placements. Reinforcement effects differentiated on the basis of the electrode used to stimulate the brain.

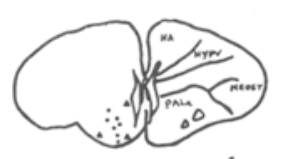

1
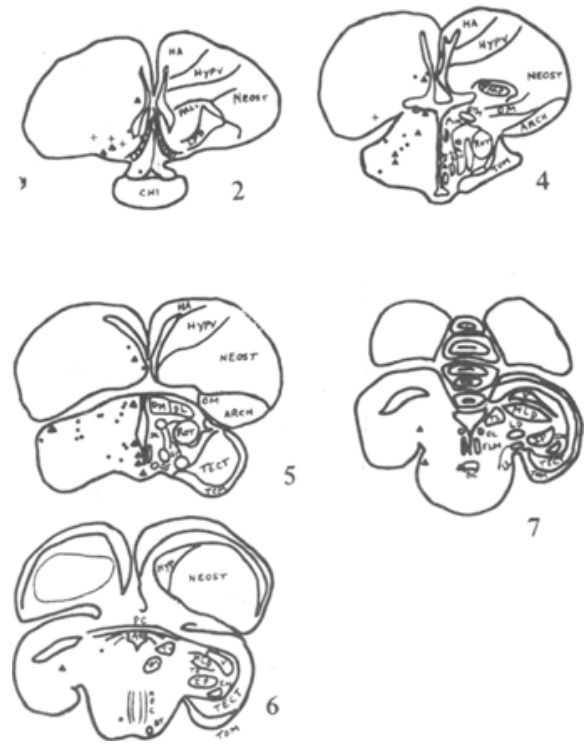

7

Fig. 2. Schematic pigeon brain cross-sections with plotted reinforcement effects. Positive reinforcement (+), negative reinforcement $\Delta$, neutral $(\cdot)$; $\mathbf{A C}$, Ant. commissure; ARCH, Archistriatum; BC, Brachium conjunctivum ; DM, Dorsomedialis nu.; LFB, Lat. forebrain bundle; MLD, mesencephalicus lat. pars dorsalis nu; NEOST, Neostriatum; PALa, Paleostriatum; ROT, Rotundus nu; TECT, Optic tectum.

\section{Histology}

A summary of the histologically identified stimulated brain points and reinforcement effects are schematically represented in Fig. 2. Positive reinforcement sites were located in the telencephalon, within the paleostriatum (three sites lateral to the descending septomesencephalic tract and one site dorsal to the anterior commissure) and in the archistriatum. Sites tested in the diencephalon and mesencephalon did not produce approach behavior.

Negative reinforcement sites were also located in the paleostriatum (in some cases adjacent to positive sites) and neostriatum. Diencephalic sites were identified in two thalamic nuclei (rotundus and dorsomedialis) and two medial hypothalamic nuclei (periventricularis magnocellularis and posterior medial). A lateral forebrain bundle placement in this region was also negatively reinforcing. In the mesencephalon, several negative points were noted within the lateral reticular formation and one in the superficial layers of the optic tectum. An apparent feature of approximately half the negatively reinforcing placements is their closeness to the brain ventricles.

Neutral points were most numerous $(57.1 \%)$ and were widely distributed throughout fore- and midbrain structures. DISCUSSION

Approach and avoidance conditioned behavior in a place-preference task was demonstrated in the pigeon, reflecting the positive and negative reinforcement effects of ESB. In addition to requiring a conditioned response differing from those employed in previously reported self-stimulation studies in the bird (Goodman \& Brown, 1966; Macphail, 1967), the present study overcame the basis for a possible argument against the validity of those findings by showing central reinforcement effects in an experimental situation not involving prior experience with another positive reinforcer, food, during training.

That the locus of stimulation was a critical factor regarding reinforcement was clearly shown by different reinforcement effects at different electrode placements within the same $S$; this finding agrees with conclusions regarding reinforcement effects seen in mammals (Olds \& Olds, 1963).

Histologies provided an interesting distribution of reinforcement points. The observation of paleostriatal positive sites supports previous findings in the pigeon (Goodman \& Brown, 1966; Macphail, 1967). Positive reinforcement in archistriatum, the avian homologue to the mammalian amygdala (Ariens-Kappers, Huber, \& Crosby, 1967), is compatible with Wurtz and Olds's (1963) findings in the rat amygdala. On the other hand. no positive sites were reportable from neostriatal. hyperstriatal, or septal ureas. Neither diencephalic nor mesencephalic points were found to be positively reinforcing, somewhat of a surprise in the light of results reported in mammals (Olds \& Olds, 1963). In response to Macphail's (1967) conclusion that the lateral forebrain bundle might represent a rostro-caudal circuit for positive reinforcement. this study is not clearly supportive in that three paleostriatal positive sites were medial to this bundle, one archistriatal positive site was lateral to it, and one diencephalic site within this bundle was negatively reinforcing.

As demonstrated in mammals (Plutchik et al, 1966; Reynolds, 1958), current intensity was also found to be a very important variable, influencing the behavior of the bird in response to ESB. Several different types of curves describing this relationship were demonstrable.

\section{REIERENCES}

ANUREW. R. J. Intracranial self-stimulation in the chick. Nature. 1967, 213.847-848.

ARIENS-KAPPERS, C. L .. HUBIE, G. C.. \& CROSBY. E. C. The comparative anatomy of the nervous s:stem of rertebrates, inchuding man. New York: Hafner. 1967.

BOYD. F.. \& GARDNER, L. Positive and negative reinforcement from intracranial self-stimulation in telcosts. Science. 1962.136. 648.

DELGADO. J. M. R., ROBERTS. W. W.. \& MILLER, N. E. Learning motivated by electrical stimulation of the brain. American Journal of Physiology, 1954. 179. 587-593.

GOODMAN. I. J.. \& BROWN, J. L. Stimulation of positively and negatively reinforcing sites in the avian brain. Life Sciences, 1966. 5. 693-704.

MACPHAIL. E. M. Positive and negative reinforcement from intracranial stimulation in pigeons. Nature, 1967, 213,947-948.

OLDS, J. \& MILNER. P. Positive reinforcement produced by electrical stimulation of the septal area and other regions of the rat brain. Joumal of Comparative \& Physiological Psychology, 1954, 47, 419-427.

OLDS. M. E.. \& OLDS, J. Approach-avoidance analysis of rat diencephalon. Journal of Comparative Veurology. 1963. 120. 259-295.

PLUTCHIK, R., MCFARLAND, W. L.. \& ROBINSON, B. W. Relationships between current intensity, self-stimulation rates, escape latencies, and evoked behavior in rhesus monkeys. Journal of Comparative \& Physiological Psychology. 1966. 61. 181-188. REYNOLDS, R. W. The relationship between stimulation voltage and rate of hypothalamic self-stimulation in the rat. Journal of Comparative \& Physiological Psychology. 1958, 51, 193-198.

WURTZ, R. H.. \& OLDS, J. Amygdaloid stimulation and operant reinforcement in the cat. Journal of Comparative \& Physiological Psychology, 1963, 56, 941-949.

$$
\text { NOTES }
$$

1. This study served as part of a dissertation submitted in partial fulfillment of requirements for the PhD degree at the lniversity of Rochester. 
2.P.H.S. Training Grant MH08034 and Research Grant MH07700 from the National Institute of Mental Health. The author acknowledges the invaluable assistance of $\mathrm{J}$. L.
Brown and R. S. Snider.

3. Present address: Psychobiology Laboratory Department of Psychology, West Virgini University, Morgantown, West Virginia 26506.

\section{Transfer of a differentiated operant ${ }^{1}$}

\author{
EDWARD J. RICKERT and KENNETH K \\ NISHIMOTO, Sacramento State College. \\ Sacramento, Calif. 95819
}

Twelve rats were trained to differentiate responses on a DRL schedule where the limited hold $(L H)$ of the schedule was either 2 or 4 sec. Two transfer groups (four Ss/group) were initially trained on LH4, while the remaining Ss were placed on $\mathrm{LH} 2$ condition. Following this training, transfer Ss were transferred either gradually or abruptly to the LH 2 condition. The results (1) indicate that the transfer of a differentiated operant parallels transfer of discrimination and (2) suggest that the major distinction between stimulus discrimination and response differentiation resides in the locus (external or internal) of the cue controlling behavior.

Two roles are commonly assigned response-produced (feedback) stimuli by a variety of learning theories. The earliest role ascribed feedback was to guide ensuing behavior; feedback from previous responding becomes a part of the stimulus complex to mediate subsequent behavior (e.g., Hull, 1930). The other role, more recently formulated, is to guide concurrent behavior; impending feedback may somehow mediate selection among alternative responses (e.g., Mowrer, 1960; Notterman \& Mintz, 1965). Rickert (1969) investigated these two roles by varying the temporal sequencing of response-produced stimulation in a discrete trial situation. i.e.. the relevant response-produced cue either preceded or occurred coincident with response selection. The results indicated that feedback shares a common property of external stimuli; namely, pretraining on an easy task facilitates learning a more difficult task.

The present research tests the generality of this finding by using another response dimension, namely, interresponse time (IRT) and a different (free-operant) procedure.

\section{METHOD}

Twelve naive female rats of Long-Evans stock, obtained from Simonsen Laboratories, served as Ss. The animals, ranging between 90 and 120 days of age at the beginning of experimentation, were housed separately, with water continuously available, and were maintained on a $12-\mathrm{g} /$ day ration for the duration of the study.

The experimental chamber was a conventional Gerbrands box (Model C) that measured $10 \frac{1}{2} \times 9-3 / 8 \times 10^{1 / 4}$ in. The intelligence panel housed a single paddle lever, located some 3 in. above the floor of the box, and a food receptacle, displaced to the left of the lever and situated $1 / 2$ in. above the box's floor, into which a 45 -mg Noyes rat pellet was delivered as re inforcement. Conventional electromechanical relay equipment controlled event sequences; impulse counters recorded responses.

Rats were shaped under continuous reinforcement until 50 reinforcements had been presented. Following shaping, a DRL schedule was imposed where the IRT was increased $1 \mathrm{sec} /$ day. When DRL 6 was attained, four Ss were assigned randomly to each of three groups: a gradual group (G), an abrupt group (A), and a difficult group (D). Rats in $G$ and $A$ groups were placed on the easy task: Reinforcement was contingent on selecting a response whose IRT equaled or exceeded 6 sec but was less than or equal to $10 \mathrm{sec}$. Group D was begun on the difficult differentiation; Ss were required to select IRTs that were $\geqslant$ 6 sec but $\leqslant 8 \mathrm{sec}$. That is to say. Groups $G$ and A were placed on a DRL6 LH4 schedule and a DRL6 LH2 was imposed on Group D. Ease of differentiation in this context presumably resided in the length of the IRT interval. Session length for all rats was $20 \mathrm{~min} /$ day.

After Groups $G$ and $A$ encountered eight sessions on the easy task, Group G was shifted to an immediate condition, i.e., DRL6 LH3, for two sessions. Following this training, both $G$ and $A$ were transferred to the difficult task for six sessions. Finally, to determine the effect of initial training, a subsequent test on the easy task was run for an additional six sessions with the three groups.

\section{RESULTS}

Figure 1 depicts the results in the form of percentage of correct responses for the three groups as a function of sessions. All bar-presses that terminated the IRT within the interval prescribed were recorded as correct responses. Inspection of the first eight sessions shows that performance of the groups is directly related to the length of the IRT interval. That an IRT interval of $4 \mathrm{sec}$ is indeed easier to differentiate than an interval of $2 \mathrm{sec}$ was confirmed by the analysis $[F(2,9)=10.60, p<.01]$.

Sessions 9-10 reflect performance of the groups after $G$ group was shifted to the immediate task. As may be seen, no real decrement ensued for $G$ group following the shift.

The crucial issue as to whether pretraining on an easy differentiation facilitates performance on a difficult differentiation is reflected in the set of curves from Sessions 11-16. An analysis of variance performed on the proportion of correct responses for the three groups summed over the six sessions on the difficult task indicated that significant differences exist among the groups $[F(2,9)=4.62, p<.05]$. These findings are consistent with the previous experiment that obtained transfer of differentiation with duration as the relevant response dimension (Rickert, 1969). In addition, the results conform to those observed with intradimensional, external stimulus shifts (e.g., Lawrence, 1952; Logan, 1966).

The last set of data points (Sessions 17.22) represents a shift by all groups to the easy differentiation, i.e., DRL6 LH4. Of interest here is whether or not Ss trained from the outset on the difficult differentiation would perform as well as Ss pretrained on the easy task.

An analysis performed on Sessions 17-22 for the three groups failed to reveal a difference between them, suggesting that Group D was learning about the task even though it was not reflected in their performance $[F(2,9)=1.49, p=n . s$.$] .$

\section{DISCUSSION}

Using an IRT interval as the relevant response dimension, S's performance on a difficult differentiation was facilitated by initial training on an easier differentiation. The results support the hypothesis that feedback stimuli obey the same laws as 